\title{
Dynamics and Causality Constraints
}

\author{
Manoelito M. de Souza \\ Departamento de Física, Universidade Federal do Espírito Santo \\ 29065-900, Vitória, ES, Brazil
}

Received on 8 October, 2001

\begin{abstract}
The physical meaning and the geometrical interpretation of causality implementation in classical field theories are discussed. Causality in field theory are kinematical constraints dynamically implemented via solutions of the field equations, but in a limit of zero-distance from the field sources part of these constraints carries a dynamical content that explains old problems of classical electrodynamics with deep implications to the nature of physical interactions.
\end{abstract}

\section{Introduction}

Causality implementation in field theory is naturally connected to the very concept of field propagation. Old and well known problems appear with a field in a close neighbourhood to its sources. Then a careful analysis is required as the kinematical constraint of a causal propagation is mixed with the dynamics of the field-source interaction. In particular, for a point-like source, there are problems with infinities and other signs of inconsistency. Thus there is a generalized belief that these infinities are consequences of the source point-size dimension and, consequently, that a point-particle cannot be regarded as a viable model for a charged elementary physical object. This, as shown in the reference [1], does not correspond to reality. The infinities associated to a point-charge self-field are consequences of the way causality has being implemented with the use of lightcones, whose vertex is a singular point; the field infinity just reflects this singularity. This work returns to the ideas raised in the reference [1] further discussing its physical and geometrical meanings. Although it is being based on the case of a point electric charge, its conclusions are of a wider generality, being valid for any theoretic framework with causality implementation (Classical and Quantum Field Theory, Quantum Mechanics, General Relativity, Statistical Mechanics, etc). It shows that the plain Maxwell's theory, in a shortdistance limit, reveals unequivocal and previously unsuspected signs of its quantum nature (the existence of photons) through the indication of a discreteness on the electromagnetic interaction, hidden behind the classical continuous formalism. It hints a proposal of a new approach, developed elsewhere [2] where fields and sources are symmetrically treated as discrete pointlike objects from which the standard continuous fields are retrieved as spacetime effective averages.
This paper is organized in the following way. The geometric vision of causality is discussed in Section II with the introduction of the new concept of extended causality in contraposition to the usual local causality and of their connection to wave-particle duality. A generalization of the standard view of causality must be noticed: the use of hypercone for describing the propagation of massive fields, characterized by non-constant proper-times. This includes the lightcone as a limiting hypercone for massless fields (constant proper-times) as in the usual approach. Section III reviews pointcharge electrodynamics in the standard (local causality) approach, pointing its inherent conflicts with a subjacent idea of extended causality. The implications of extended causality to field-source dynamics is exposed in Sections IV and V. The paper closes in Section VI with some generic comments about the characteristics and consequencess of a formulation of field theory in terms of discrete interactions.

\section{Causality and spacetime geometry}

The notation used is of omitting the spacetime indices when this causes no ambiguity. For example, $\partial$ for $\partial_{\mu}$, and $A(x, \tau)$ for a vector field $A^{\mu}(x, \tau) ; x$ stands for both, the event parameterized by $x^{\mu}=(t, \vec{x})$ and for the coordinate $x^{\mu}$ itself.

Any given pair of events on Minkowski spacetime defines a four-vector $\Delta x$. In our notation, therefore, $\Delta x$ is not meant to be necessarily an infinitesimal, for which we make use of $d x$. The propagation of a free massless field on a flat spacetime of metric $\eta_{\mu \nu}=$ $\operatorname{diag}(-1,1,1,1)$, is restricted by

$$
\Delta x^{2}=0
$$


which defines a local double (past and future) lightcone containing this four-vector $\Delta x: \Delta t= \pm|\Delta \vec{x}|$. This is also a mathematical expression of local causality in the sense that it is a restriction for the massless field to remain on this lightcone. It is a particular case of the more generic expression

$$
\Delta \tau^{2}=-\Delta x^{2}
$$

which is, besides, the definition of the proper time $\tau$ associated to the propagation of a free physical object across $\Delta x$. As $\tau$ is a real valued parameter, the Eq. (2) just expresses that $\Delta x$ cannot be spacelike. Geometrically it is also the definition of a three-dimensional double hypercone, of which the lightcone and the time axis are just the two extreme limiting cases. $\Delta x$ is the four-vector separation between a generic event $x$ and the hypercone vertex. This conic hypersurface is the support for the definition of a propagating field. The hypercone aperture-angle $\theta, 0 \leq \theta \leq \pi / 4$, is given by $\tan \theta=\frac{|\Delta \vec{x}|}{|\Delta t|}, c=1$, or $\Delta \tau^{2}=(\Delta t)^{2}\left(1-\tan ^{2} \theta\right)$. A change of the supporting hypercone corresponds to a change of speed of propagation and is an indication of interaction.

Let us consider a field on a point $x$ of a hypercone defined by the Eq. (2). Therefore we know $\Delta x$, the fourvector determined by $x$ and by its past-hypercone vertex on its source's wordline. Continuity requirements of the field propagation lead us to apply the constraint (2) on a neighbourhood of $x:(\Delta \tau+d \tau)^{2}=-(\Delta x+d x)^{2}$ or, after using Eq. (2), $\Delta \tau d \tau+\Delta x . d x=0$, which may be written as

$$
d \tau+f . d x=0,
$$

where $f$ is a constant four-vector tangent to the hypercone (2), entirely defined by $\Delta x$ (free propagation). For $\Delta \tau \neq 0$ it is just

$$
f^{\mu}:=\left.\frac{\Delta x^{\mu}}{\Delta \tau}\right|_{\Delta \tau^{2}+\Delta x^{2}=0}
$$

For $\Delta \tau=0$ the hypercone (2) reduces to the lightcone (1) and $f$ to its tangent four-vector along $\Delta x ; f$ and $\Delta x$ are both lightlike. It is important to observe that $f$ is well defined for any $\Delta \tau$, including $\Delta \tau=0$, as long as $\Delta x \neq 0$. A tangent is not defined at a hypercone vertex. This is a crux point, neglected in the existing literature $[3,4,5,6,7,8]$ which leads to the old and well known vexing problems of consistency in classical electrodynamics [1]. Geometrically Eq. (3) defines a hyperplane tangent to the hypercone (2). The simultaneous imposition of Eqs. (2) and (3) on the propagation of a free point object produces a much more stringent constraint than local causality as the object is restricted to remain on the intersection of the hypercone (2) with its tangent hyperplane (3), that is, on the hypercone generator tangent to $f$, or the $f$-generator, for short. It assures that $d x$ of Eq. (3) is always collinear to $\Delta x$.
Or, in other words, that a free pointlike object propagates on a straightline (which is tangent to $f$ ), exactly as required by Newton's first law. This corresponds to an amplified concept of causality which will be referred to as extended causality.

Local and extended causality correspond to two distinct and complementary (like geometric and wave optics) description of a same physical system. They correspond to different perceptions of the spacetime available to the free evolution of a physical system from a given initial condition, respectively as foliations of hypercones and as congruences of straight lines, the hypercone generators. So, whereas the first one is appropriated for a description in terms of continuous and extended objects like a fluid, a field, a wave, the second one implies on a perception of them as discrete sets of points, describing individually each point.

\section{Point-charge electrodynam- ics}

It is worthwhile to review the standard approach for the point-charge electrodynamics vis-à-vis the extended causality concept. Consider, for example, $z(\tau)$, the worldline of a classical point electron parameterized by its proper time $\tau$; each event on this worldline belongs to the (instantaneous) hypercone

$$
\Delta \tau^{2}+\Delta z^{2}=0
$$

where, again, the four-vector $\Delta z$ is defined by the event $z$ and the vertex of a hypercone (not a lightcone, for a massive electron) that passes by $z$; the four-vector $u=\frac{d z}{d \tau}$ is tangent to the worldline (and to the local hypercone). It satisfies

$$
d \tau+u . d z=0
$$

which corresponds to Eq. (3). A free electron remains on the $u$-generator of its hypercone; an accelerated electron is on a $u$-generator of its instantaneous hypercone. So, in a way, classical electrodynamics already uses extended causality for specifying the state of the classical electron, and this is consistent with an electron modeled as a point particle. Let us discuss how extended causality enters in the definition of the electromagnetic field in a conflictive way. It hints to a new consistent formulation for field theory.

Consider now the electromagnetic field at $x$, emitted by this electron. $\Delta x=x-z(\tau)$ defines a family of four-vectors connecting the event $x$ to events on the electron worldline $z(\tau)$. Then, accounting for the masslessness of the electromagnetic field, $\Delta x^{2}=0$, the double lightcone with vertex at $x$, intercepts $z(\tau)$ at two points: $z\left(\tau_{\text {ret }}\right)$ and $z\left(\tau_{a d v}\right)$. See the Fig. 1 . 


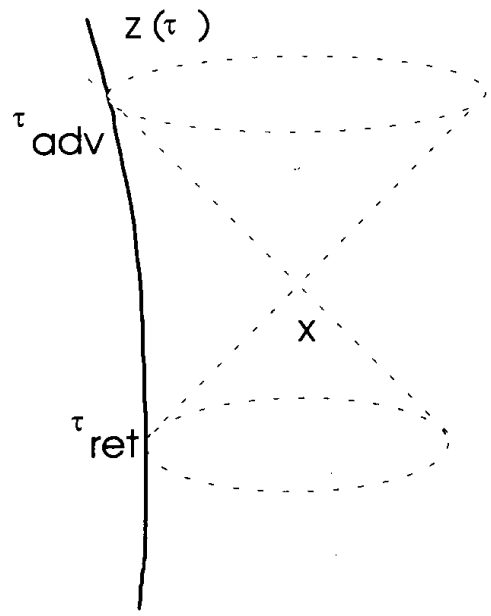

Figure 1. The advanced and the retarded Liènard-Wiechert fields at an event $\mathrm{x} . \tau_{a d v}$ and $\tau_{\text {ret }}$ are the two intersections of the double hypercone $\Delta x^{2}=0$, for $\Delta x=x-z(\tau)$, with the electron worldline $z(\tau)$.

The retarded field emitted by the electron at $z\left(\tau_{\text {ret }}\right)$ must remain in the $z\left(\tau_{\text {ret }}\right)$-future-lightcone, which contains $x$; and according to the standard interpretation $[3,4,5]$, the advanced field produced by the electron at $z\left(\tau_{a d v}\right)$ must remain in the $z\left(\tau_{a d v}\right)$-past-lightcone, which also contains $x$. So, the electromagnetic potential field is defined just with local causality. There is then a clear dichotomy with respect to causality implementation in the treatment done to the electron and to its self-field [9], caused by the perception of the electron as a point particle, a discrete object, and of its field as a continuous and distributed one. Extended causality requires and implies discrete objects.

The (retarded and advanced) Liènard-Wiechert solutions of classical electrodynamics $[3,4,5]$ are

$$
A^{\mu}(x)=\left.\frac{e u^{\mu}(\tau)}{\rho}\right|_{\tau=\tau_{s}}, \quad \text { for } \rho \neq 0,
$$

where $\tau_{s}$ stands for either $\tau_{r e t}$ or $\tau_{a d v}$, which are, re- spectively, the retarded and the advanced solution to the constraint

$$
(x-z(\tau))^{2}=0,
$$

imposed to $A(x)$, and

$$
\rho:=-u \cdot \Delta x,
$$

with $\Delta x=x-z(\tau)$, represents $|\Delta \vec{x}|$ in the charge restframe. Although $A(x)$ is restricted just by Eq. (1), having thereby support on the lightcone, for the calculation of its Maxwell field

$$
F_{\mu \nu}:=\partial_{\nu} A_{\mu}-\partial_{\mu} A_{\nu}
$$

on a point $x$ it is necessary to consider $A(x)$ on a neighbourhood of $x$, and so a constraint equivalent to the Eq. (3) must be also considered to assure the consistency of Eq. (1) in this neighbourhood. From Eq. (8) one has

$$
\Delta x \cdot d(x-z)=\Delta x \cdot(d x-u d \tau)=0,
$$

which leads to

$$
d \tau+K . d x=0
$$

where $\mathrm{K}$ defined by

$$
K^{\mu}=\left.\frac{\Delta x^{\mu}}{-u . \Delta x}\right|_{\tau_{s}}=\left.\frac{\Delta x^{\mu}}{\rho}\right|_{\tau_{s}}
$$

is a null $\left(K^{2}=0\right)$ four-vector, tangent to the lightcone $\Delta x^{2}=0 . K^{\mu}$ shows the local direction of propagation of the electromagnetic field emitted by the electron at $\tau_{s}$. In this context, Eq. (11) is a consistency relation of Eq. (8), assuring its validity for all successive pair of events $(x, z(\tau))$. It implies on

$$
K_{\mu}=-\frac{\partial \tau_{s}}{\partial x^{\mu}}
$$

where $\tau_{s}$, a solution of Eq. (8), is seen as a function of $x$. Then,

$$
-\left.\frac{1}{e} \partial_{\mu} A^{\nu}\right|_{\tau_{s}}=\left.\left\{\frac{K_{\mu} a^{\nu}}{\rho}+\frac{u^{\nu}}{\rho^{2}} \partial_{\mu} \rho\right\}\right|_{\tau_{s}}=\left.\frac{1}{\rho^{2}}\left(K_{\mu} W^{\nu}+u_{\mu} u^{\nu}\right)\right|_{\tau_{s}}
$$

where $a:=\frac{d u}{d \tau}$,

$$
\left.\partial_{\mu} \rho\right|_{\tau_{s}}=\left.\left\{K_{\mu}(1+\rho a . K)-u_{\mu}\right\}\right|_{\tau_{s}}
$$

and the ancillary four-vector function $W$,

$$
W^{\mu}=\left.\left\{\rho a^{\mu}+u^{\mu}(1+\rho a . K)\right\}\right|_{\tau_{s}},
$$

has been introduced just for notation simplicity. So,

$$
F^{\mu \nu}=\left.\frac{1}{\rho^{2}}\left(K^{\mu} W^{\nu}-K^{\nu} W^{\mu}\right)\right|_{\tau_{s}}
$$

Geometrically the Eq. (11), like the Eq. (3), defines a family of hyperplanes that, for $d \tau=0$, are tangent to the lightcone (8), and are parameterized by 
$K_{\mu}=\eta_{\mu \nu} K^{\nu}$. The use of both constraints (8) and (11) singles out the light-cone generator tangent to $K^{\mu}$ and inserts extended causality in the $F$ definition, which is exhibited on its explicit dependence on $K^{\mu}$. But rigourously this is an inconsistent procedure as an undue mixing of local and extended causality on a same physical object. The inconsistency is on $F$ being defined as the curl of $A(x)$ which is a continuous field with support on the lightcone. If its support is reduced to the $K$-generator of its lightcone, $F$ has to be regarded as a discrete object, similar, in this aspect, to its very source, the point electron. The problem, of course, is not with the definition (10) of $F$ but with $A(x)$ being a propagating extended field and, therefore, restricted by a causality constraint that necessarily requires the constraint (11) on any field derivative. In other words, there would be no problem with the definition (10) if $A(x)$ where not constrained by (8) because the constraint (11) would not be called up then. Since the constraint (11) cannot be avoided a completely consistent formulation requires $A(x)$ being defined with extended causality too. This leads to the consideration of fields defined with support on $(1+1)$ submanifolds imbedded in the $(3+1)$ spacetime, "discrete fields", with a complete symmetry between fields and sources; both being discrete objects. This is done in the reference [2]. The goal of the present paper is just of pointing the existence of two modes for causality implementation (local and extended) in field theory and their implications to the meaning and nature of the fields and of their interactions.

In the standard literature, without knowledge of extended causality, $F$ is seen as a field with support on the lightcone, i.e. as a continuously extended object and, then, with the old and well-known problems with infinities and other inconsistencies. These problems just vanish after due consideration of extended causality [1].

The origin of this imbroglio is that the Equation (11), as it can be formally obtained from a derivation of Eq. (8), has been historically considered [3, 4, 5] as if all its effects were already described by Eq. (8), included in it and not, as it is the case, a new and independent restriction to be considered at a same footing and in addition to it. An evidence of this is that Eqs. (8) and (11) carry distinct physical informations as we discuss now.

\section{Dynamics and causality}

Eq. (11) connects the restriction on the propagation of the charge to the restriction on the propagation of its emitted or absorbed fields. Like its parent Eq. (8) it is just a kinematical restriction. But in the short-distance limit, when $x$ tends to $z(\tau)$, Eq. (11), in contradistinction to Eq. (8), is directly related to the changes in the charge's state of movement due to the emission or to the absorption of electromagnetic field, that is, to the charge-field interaction process. Therefore, in this short distance limit Eq. (11) also carries dynamical information, not only kinematical, as is the case of Eqs. (5) and (8).

It is instructive to have a close look on the physical meaning of Eqs. (8) and (11) for the case of an emitted field. Eq. (8) is a restriction on the propagation of a single object, the field emitted by the charge at $z(\tau)$, whereas the Equation (11) connects restrictions on the propagation of two distinct physical objects, the electron and its field: $d \tau$ describes a displacement of the electron on its worldline while $d x$ is the four-vector separation between two other points where the electron self-field is being considered. If $d \tau=0$ then $d x$ is lightlike and collinear to $\mathrm{K}$, as $K . d x=0$. Thus, $d x$ is related to a same electromagnetic signal at two distinct times. The electromagnetic field at $x+d x$ can be seen as the same field at $x$ that has propagated to there with the speed of light. On the other hand, if $d \tau \neq 0$ then $d x$ is not collinear to $\mathrm{K}$ and it is related to two distinct electromagnetic signals, emitted at distinct times. See the Fig. 2. In this case, the field at $x+d x$ cannot be seen as the same field at $x$ that has propagated to there. It is another field emitted by the charge at another time. This apparently obvious interpretation of the constraint (11) reveals, however, deep physical implications as it perceives as being distinct objects the fields $F$ in two events that are not along a same four-vector $K$. This comes from extended causality requiring a $F$ defined with support on a $K$-light-cone generator and conflicting with local causality in the definition of $A(x)$.

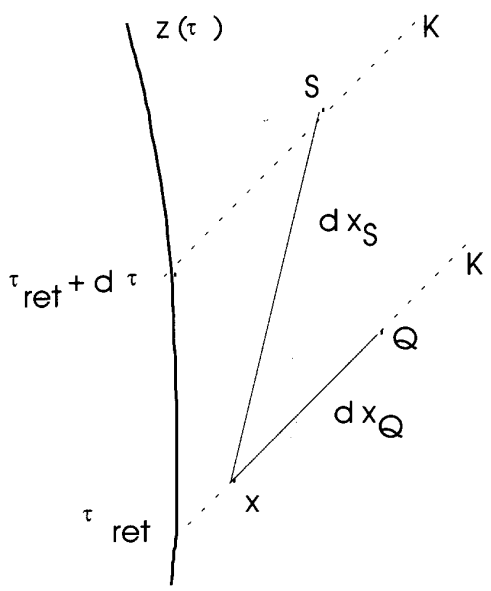

Figure 2. The field at the point $\mathrm{Q}$ may be considered as the same field at $x$ that has propagated to Q, because $d x_{Q}$ is collinear to K. The fields at events $x$ and S are two distinct signals emitted by the charge at two distinct times $\tau_{r e t}$ and $\tau_{\text {ret }}+d \tau$ as $d x_{S}$ is not collinear to $\mathrm{K}$.

But a $F$ defined with support on a lightcone generator produces strong and experimentally observable consequences. The Eq. (11) implies on

$$
1+K \cdot u=0 \text {. }
$$


During the free propagation of an electromagnetic radiation, the four-vector $K$ of its light-cone-generator support must be constant. This is in contradistinction to the standard (local causality) approach where $K$, defined by Eq. (12), is a continuous function of the spacetime point. With extended causality Eq. (11) is replaced by Eq. (3) as $K$ is replaced by $f$ which by construction is a constant four-vector defined by $\Delta x$, according to Eq. (4). Then, from a derivation of Eq. (18), (with $f$ replacing $k$ )

$$
f . a=0 .
$$

$1+f . u=0$ may be seen as a covariant normalization of $f$, that in the charge instantaneous rest frame must satisfy

$$
\left.f^{0}\right|_{\vec{u}=0}=\left.|\vec{f}|\right|_{\vec{u}=0}=1 .
$$

The Eq. (19) is a dynamical constraint between the direction $f$ along which the signal is emitted (absorbed) and the instantaneous change in the charge state of motion at the retarded (advanced) time. It implies on

$$
a_{0}=\frac{\vec{a} \cdot \vec{f}}{f_{0}},
$$

whereas $a . u \equiv 0$ leads to

$$
a_{0}=\frac{\vec{a} \cdot \vec{u}}{u_{0}}
$$

and so, in the charge instantaneous rest frame at the limiting emission (absorption) time $\vec{a}$ and $\vec{f}$ are orthogonal vectors,

$$
\left.\vec{a} \cdot \vec{f}\right|_{\vec{u}=0}=0 .
$$

This is an observable consequence of extended causality. For the electromagnetic field this is an old well known and experimentally confirmed fact $[10,11,13]$. The demonstration, in the standard formalism of continuous fields, that "radiation is emitted only in a direction orthogonal to a non-vanishing component of acceleration" takes [11] the whole apparatus of Maxwell's theory. With extended causality it can be demonstrated on very generic grounds of causality without reference to any specific interaction. Its experimental confirmation validates extended causality and makes of it a universal relation, supposedly valid for all kinds of fields and sources. This same behaviour, expressed in Eq. (21), is then expected to hold for all fundamental (strong, weak, electromagnetic and gravitational) interactions. The standard formalism deals with fields as continuous and extended objects, like a wave, and so their direction of propagation $K$ is, necessarily a local function, even for free fields on a flat background spacetime. In contradistinction, extended causality deals with discrete field for which there is a single constant direction of free propagation. The concept of a discrete radiation as a photon is natural and inherent, right after its emission (or right before its absorption) and does not require taking the field largedistance limit as in the usual approach.

\section{A. The hypothesis of $f$ being constant}

The relevance of Eq. (19) is on its focus on the charge-field interaction process. It is strongly dependent on $f$ being taken as a constant during the field propagation which implies free propagation between consecutive discrete interaction events. A non-constant $f$, and so here it is being replaced back to $K$, would imply on a continuing interaction and this would change the above results. From Eqs. (12) and (15),

$$
\partial_{\mu} K_{\nu}=\frac{1}{\rho}\left(\eta_{\mu \nu}+K_{\mu} u_{\nu}+K_{\nu} u_{\mu}-K_{\mu} K_{\nu}\right)-K_{\mu} K_{\nu} a . K=\partial_{\nu} K_{\mu}:=K_{\mu \nu} .
$$

Then the hypothesis of a non-constant $K$ would not affect Eq. (18) because

$$
K_{\mu \nu} \Delta x^{\nu}=\rho K_{\mu \nu} K^{\nu}=K_{\mu}(1+K . u) \equiv 0,
$$

but Eq. (19) would be replaced by just an identity as

$$
\nabla_{\mu}\left(1+K_{\nu} u^{\nu}\right)=K_{\mu \nu} u^{\nu}-K_{\mu} K_{\nu} a^{\nu}=K_{\mu}(1+K . u) \equiv 0 .
$$

So, it is clear that the validity of Eq. (19) rests on a free propagation of the field right after its emission (or, symmetrically, right before its absorption) which indicates no self-interaction, a definitive detachment of the field from its source. Self interaction for the emitted field would also imply, by symmetry, causality violation for the absorbed field as it would be interacting with the charge even before reaching it. 


\section{The double simultaneous limit}

The above conclusions can be made more evident considering the fate of both Eqs. (2) and (11) in the limit when the event $x$ approaches the event $z\left(\tau_{s}\right)$ and its implications to the field energy-tensor ${ }^{1}$. Nothing obviously happens to the first one; $\Delta x$ just goes to zero. To the second one the restriction connecting $d \tau$ to $d x$ becomes indeterminated because $\mathrm{K}$ is not defined in this limit:

$$
\lim _{x \rightarrow z\left(\tau_{s}\right)} K=\lim _{x \rightarrow z\left(\tau_{s}\right)} \frac{\Delta x}{-u \cdot \Delta x}=\frac{0}{0} ?
$$

For a lightlike signal, Eqs. (2) and (11) together require that the pair of events $x$ and $z(\tau)$ belongs to a same lightcone generator, so that Eq. (25) can be written as

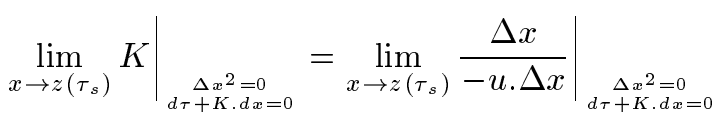

This notation intends to denote that $x$ approaches $z\left(\tau_{s}\right)$ through a K-light-cone generator, i.e. by the straight line intersection of the hypercone $\left(\Delta x^{2}=0\right)$ and its tangent hyperplane $(d \tau+K \cdot d x=0)$, eliminating any ambiguity in the definition of the limit in Eq. (25). Now one can apply the L'Hôpital's rule for evaluating $\mathrm{K}$ on the neighbouring events of $z\left(\tau_{s}\right)$ along the electron worldline, i.e., at either $\tau_{s}+d \tau$ or $\tau_{s}-d \tau$. This corresponds to replacing the above simple limit of $x \rightarrow z\left(\tau_{s}\right)$ by a double and simultaneous limit of $x \rightarrow z(\tau)$ along the K-lightcone generator while $z(\tau) \rightarrow z\left(\tau_{s}\right)$ along the electron worldline. This simultaneous double limit is pictorially best described by the sequence of points $\mathrm{S}$, $\mathrm{Q}, \ldots, \mathrm{P}$ in the Fig. 3; each point in this sequence belongs to a K-generator of a lightcone with vertex at the electron worldline $z(\tau)$.

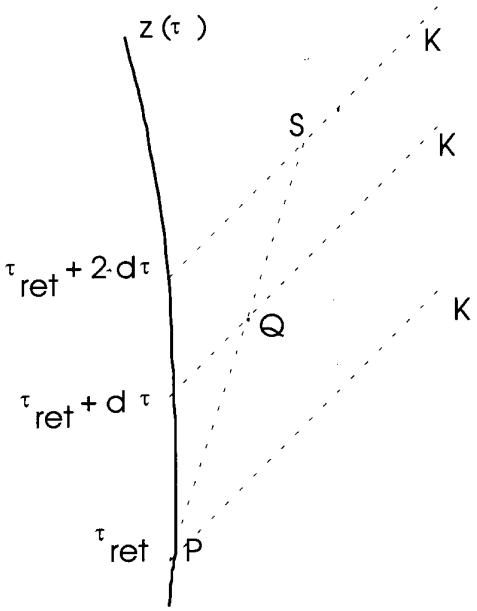

Figure 3. The double limit $x \rightarrow z\left(\tau_{\text {ret }}\right)$ along the SQ...P line consists of $x \rightarrow z(\tau)$ along the light-cone generator $\mathrm{K}$ while $\tau \rightarrow \tau_{\text {ret }}$ on the electron worldline.

Then, from Eq. (25),

$$
\begin{gathered}
\left.\lim _{\substack{x \rightarrow z(\tau) \\
\tau \rightarrow \tau_{s}}} K\right|_{\substack{\Delta \tau+K . d x=0 \\
d x^{2}=0}}=\left.\lim _{\substack{x \rightarrow z(\tau) \\
\tau \rightarrow \tau_{s}}} \frac{\Delta \dot{x}}{-(a . \Delta x+u \cdot \Delta \dot{x})}\right|_{\substack{\Delta, \Delta x^{2}=0 \\
d \tau+K \cdot d x=0}}= \\
=\left.\lim _{\substack{x \rightarrow z(\tau) \\
\tau \rightarrow \tau_{s}}} \frac{-u}{u \cdot u}\right|_{\substack{\Delta x^{2}=0 \\
d \tau+K . d x=0}}=u,
\end{gathered}
$$

as $\Delta \dot{x}:=\frac{d \Delta x}{d \tau}=-\frac{d z}{d \tau}=-u$ and $u^{2}=-1$. So $\left.K\right|_{x=z\left(\tau_{s}\right)}$ is indefinite but $\left.K\right|_{x=z\left(\tau_{s} \pm d \tau\right)}=u$.

The lightlike four-vector $\mathrm{K}$ is replaced by the timelike four-vector $\mathrm{u}$ in the above defined (double) limit of $\Delta x \rightarrow 0$. The limit (27) is straightforward and has very transparent geometrical and physical interpretations. Its validity cannot be discussed. This remark must be made because Eq. (27) radically changes the usual vision of field theory in the short-distance limit.

The electron self-field energy tensor, $4 \pi \Theta=F . F-\frac{\eta}{4} F^{2}$, after Eq. (14) becomes

$$
-4 \pi \rho^{4} \Theta=(K W+W K)+K K W^{2}+W W K^{2}+\frac{\eta}{2}\left(1-K^{2} W^{2}\right),
$$

as $K . u=-1$ from Eq. (12) and $K . W=-1$. The Eq. (28), like Eqs. (7) and (17), are all constrained by Eq. (2), i.e. by $\tau=\tau_{s}$, and they are valid only for $\rho \neq 0$, region where $K^{2}=0$. So, instead of Eq. (28) one may write

$$
-\left.4 \pi \rho^{4} \Theta\right|_{K^{2}=0}=(K W+W K)+K K W^{2}+\frac{\eta}{2}, \quad \text { for } \quad \rho>0, \tau=\tau_{s},
$$

\footnotetext{
${ }^{1}$ This is discussed in reference [1] but for completeness, considering its relevance here, its main steps and some further considerations are aligned.
} 
which corresponds to the usual expressions found in the literature $[3,4,5,6,7,8]$. They are equivalent, as long as $\rho>0$. The four-vector momentum associated to the electron self-field is defined by the flux of its $\Theta$ through a hypersurface $\sigma$ of normal n:

$$
P=-\int d^{3} \sigma n .\left.\Theta\right|_{K^{2}=0},
$$

but $\Theta$ contains a factor $\frac{1}{(\rho)^{4}}$ and this makes $\mathrm{P}$ highly singular at $\rho=0$, that is at $x=z\left(\tau_{s}\right)$. This is the old well-known self-energy problem of classical electrodynamics which heralds [12] similar problems in its quantum version. This divergence at $\rho=0$ is also the origin of nagging problems on finding a classical equation of motion for the electron $[3,4,5,6,7]$. But it is clear now, after Equation (27), that the standard practice of replacing everywhere $\Theta$ by $\left.\Theta\right|_{K^{2}=0}$ is not justified and, more than that, it is the cause of the above divergence problem and the related misconceptions in classical electrodynamics. One must use Eq. (28), the complete expression of $\Theta$, in Eq. (30) and repeat for it the same double limit done in Eq. (27). The long but complete and explicit calculation is done in reference [1]; its results and conclusions are summarised here:

$\left.P\right|_{x=z\left(\tau_{s}\right)}$ is undefined but

$$
\left.P\right|_{x=z\left(\tau_{s}-\right)}=\left.P\right|_{x=z\left(\tau_{s}+\right)}=0 .
$$

There is no infinity at $\rho=0$ ! This infinity disappears only when the double limiting process is taken because the lightcone generator $K$ must then be recognized as the actual support of the Maxwell field $F$. The message here is that the infinities and other inconsistencies of classical electrodynamics are not to be blamed on the point electron but on the lightcone support of the field in the Eq. (7). Extended causality cannot be ignored in a finite and consistent field theory.

\section{Conclusions}

We can summarize it all with the following implications to the charge-field dynamics:

1. No self interaction. After its emittion the field no longer interacts with its source.

2. The emission/absorption process is discrete.

3. The emission/absorption event is an isolated singularity on the charge worldline; singular in the sense of discontinuity on its first derivative. See the Fig. 4.

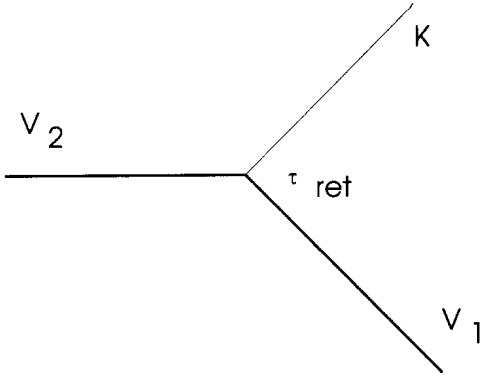

Figure 4. The sudden change in the electron state of movement and either its cause (the absorption of a photon, for example) or its consequence (the emission of a photon). There is no electron self-field immediately before or after $\tau_{\text {ret }}$. It is an isolated singularity. $\tau_{\text {ret }}$ is a singular point on the electron wordline only because its tangent is not defined there; there is no infinity.

Equation (31) confirms that $z\left(\tau_{s}\right)$ is an isolated singularity. This is in direct contradiction to the standard view of a classical continuous field, emitted or absorbed by the charge in a continuous way. According to Eq. (31) there is no charge self field at $z\left(\tau_{s} \pm d \tau\right)$, but only sharply at $z\left(\tau_{s}\right)$. It is saying that the Gauss' law, in the zero-distance limit,

$$
\lim _{S \rightarrow 0} \int_{S} d \sigma \vec{E} \cdot \vec{n}=4 \pi e
$$

is meaningful (in terms of an effective continuous field) only at $z\left(\tau_{s}\right)$ and not at $z\left(\tau_{\text {ret } \pm d \tau}\right)$ because $\vec{E}\left(\tau_{s}\right) \neq 0$ but $\vec{E}\left(\tau_{r e t \pm d \tau}\right)=0$.

It implies, in other words, that the electromagnetic interactions are discrete and localized in time and in space. In terms of a discrete field interaction along a lightcone generator, as the one represented in the Fig. 4, one can understand the physical meaning of Eqs. (26), (27) and (31). The continuous Maxwell fields are just effective average descriptions of an actually discrete interaction field. The field discreteness (or the existence of photons) is masqueraded by this averaged field and it takes the zero-distance limit to be revealed from the Maxwell field. It is remarkable that these conclusions have been derived exclusively from the supposedly exhaustively known classical electrodynamics but nothing has been added to or modified in the old Maxwell's theory, except a new interpretation of old well known results. They come from the recognition of the existence of two mutually excluding causality-implementation modes in the Maxwell's formalism. This could have been taken, at the beginning of the last century, as a first indication of the quantum, or of the discrete nature of the electromagnetic interaction. They are all consequences of the dynamical constraints hidden on the restrictions (2) and (11).

The initial goal of discussing the implicit existence of two distinct modes of implementing causality in field theory has been fulfilled. A completely consistent field formalism must be expressed in term of fields defined 
ab initio with extended causality. How this can be accomplished, its consequences and how it is related to the standard formalism based on local causality is discussed in reference [2].

The Fig. represents the sudden change in the electron state of movement and either its cause (the absorption of a photon, for example) or its consequence (the emission of a photon).

\section{References}

[1] M. M. de Souza, "Classical Electrodynamics and the Quantum Nature of Light". J. of Phys A: Math. Gen. 30 (1997)6565-6585. hep-th/9610028

[2] M. M. de Souza, Conformally symmetric massive discrete fields. Hep-th/0006237.

[3] F. Rorhlich Classical Charged Particles. Reading, Mass. (1965).
[4] D. Jackson Classical Electrodynamics, 2nd ed., John Wiley \& Sons, New York, NY(1975).

[5] S. Parrot, Relativistic Electrodynamics and Differential Geometry, Springer-Verlag, New York, 1987.

[6] C. Teitelboim; D. Villaroel; Ch.G.Van Weert, Rev. del Nuovo Cim., vol 3, N.9, (1980).

[7] E.G.P. Rowe, Phys. Rev. D 12, 1576 (1975) and 18, 3639 (1978); Nuovo Cim. B 73, 226 (1983).

[8] A. Lozada, J. Math. Phys. 30, 1713 (1989).

[9] M. M. de Souza, Braz. J. of Phys. 28, 250 (1999). Hepth/9505169

[10] See, for example, chapter 14 of reference [4].

[11] See, for example, the reference [3] at page 112.

[12] J. Schwinger in Selected papers on Quantum Electrodynamics, J. S. Schwinger (ed.), p. XV-XVIII, Dover, New-York, NY,(1958).

[13] A.A. Sokolov, I. M. Ternov, Radiation from relativistic electrons, A.I.P, New York, N. Y, 1986. 